\title{
PROTO-PLANETARY NEBULAE AS EXPLOSIONS: BULLETS VERSUS JETS AND NEBULAR SHAPING
}

\author{
Timothy J. Dennis, ${ }^{1}$ Andrew J. Cunningham, ${ }^{1}$ Adam Frank, ${ }^{1}$ \\ Bruce Balick, ${ }^{2}$ Eric G. Blackman, ${ }^{1}$ and Sorin Mitran ${ }^{3}$ \\ Received 2007 July 11; accepted 2008 February 20
}

\begin{abstract}
Many proto-planetary nebulae (PPNs) appear as narrow collimated structures sometimes showing multiple, roughly aligned lobes. In addition, many PPN flows have been shown to have short acceleration times. In this paper we explore whether jet or "bullet" (a massive clump) models fit the observations of individual collimated lobes adequately by comparing simulations of both radiatively cooled (stable) jets and bullets. We find that the clump model is favored over jets because (1) it leads to greater collimation of outflows, (2) it accounts better and more naturally for ringlike structures observed in the PPN CRL 618, and (3) it is more successful in reproducing the Hubble-flow character of observed kinematics in some PPNs. In addition, bullets naturally account for observed multipolar flows, since the likely MHD launch mechanisms required to drive outflows make multiple nonaligned jets unlikely. We also find that the bow shock heads of bullets take on a V-shaped configuration, whereas bow shock heads of jets are more U-shaped. The differences in these configurations occur on a linear scale corresponding to an angular size of the order of $\sim 3^{\prime \prime}$ — sufficiently large to suggest a viable means of distinguishing bullets from jets in observations. We argue that PPN outflows may be driven by explosive MHD launch mechanisms such as those discussed in the context of supernovae (SNe) and gamma-ray bursts (GRBs).
\end{abstract}

Subject headings: ISM: jets and outflows — planetary nebulae: general — planetary nebulae: individual (CRL 618) — stars: AGB and post-AGB

\section{INTRODUCTION}

In the past decade, images of very young planetary nebulae (PNs) and proto-planetary nebulae (PPNs) have revealed an unexpected diversity of morphological classes. Many of these objects appear to exhibit a level of complexity that cannot be accounted for in terms of the generalized interacting stellar winds model (GISW; Balick \& Frank 2002 and references therein). Of particular interest are objects exhibiting point-symmetric, multipolar, and "butterfly" morphologies, as well as bipolar and multipolar objects exhibiting highly collimated "jetlike" outflows. We present examples of some multipolar objects in Figure 1.

The appearance of these collimated and sometimes multipolar outflows in so many PPNs has led to the suggestion that highspeed jets operate during the late asymptotic giant branch (AGB) and/or post-AGB evolutionary phases of the central star (Sahai \& Trauger 1998). While the GISW model can account for narrow jets (Icke et al. 1992; Mellema \& Frank 1997; Borkowski et al. 1997), it assumes the winds are radiatively driven. Radiative acceleration cannot, however, account for these flows, since a number of observational studies demonstrate a momentum excess such that a factor of $\sim 10^{3}$ exists between outflow momentum observed and what can be attributed to stellar radiation pressure (Bujarrabal et al. 2001). Moreover, it is difficult to attribute the degree of observed collimation to a large-scale dust torus as is usually required in the GISW model. In addition, the problem of accounting for the precession necessary for the production of point-symmetric flows remains to be solved (for an accretion-disk-based model see Icke 2003). For these reasons, the suggestion has been made

\footnotetext{
1 Department of Physics and Astronomy, University of Rochester, Rochester, NY 14627; tdennis@pas.rochester.edu.

2 Department of Astronomy, University of Washington, Seattle, WA 98195.

3 Department of Mathematics, University of North Carolina, Chapel Hill, NC 27599.
}

that PPN jets and collimated outflows are magnetically driven (Blackman et al. 2001a, 2001b; Frank \& Blackman 2004; Matt et al. 2006; Frank 2006). Magnetically driven models couple rotation to a magnetic field. Jets therefore are bound to flow along the rotational axis of the central object, and it is difficult to see how multiple jets of similar size can be driven by such a mechanism. We discuss these models and this issue in more detail at the end of the paper.

Observationally, clumps and collimated flows occur in many stellar outflows, although not always together. The outflows in Wolf-Rayet (WR) nebulae are clumpy, but jets are not observed. In young stellar objects (YSOs), jets and collimated bipolar outflows are quite common, and while they can often be clumpy, the jet beams - distinct from the bow shocks which they drive - are often apparent, stretching all the way back to the stellar source. In mature PNs, clumps are often seen (as in NGC 2392 [Eskimo], NGC 6853 [Dumbbell], and NGC 7293 [Helix]). Fully articulated jets, however, are very rare. We note that ionization shadows and "mass loaded" flows behind clumps can give the appearance of jets. In some mature PNs such as the Cat's Eye Nebula, structures appear (some of which fall under the term "FLIERs") that may be the remnants of poleward-directed flows. In Hubble Space Telescope images of many PPNs, the outlines of reflected light are often bipolar, but within these boundaries the illuminated gas seems irregular. Thin jets (as opposed to thin finger-shaped lobes) are rarely seen directly except (perhaps) in $\mathrm{OH} 231.8+4.2$ (Calabash). However, pairs or sets of knots lying along or near the apparent symmetry axes are not unusual (M1-92, IRAS 20028+ 3910 , IRAS 16594-4656, Hen 3-1475). Thus, the creation of continuous jets as in the case of YSOs does not seem to be the norm in PNs and PPNs.

Clumps or "bullets" driven into the surrounding media have been found to be an effective explanation for some stellar outflow structures. In Poludnenko et al. (2004) the authors modeled the strings of $\eta$ Car as bullets of high-speed material ejected by the 


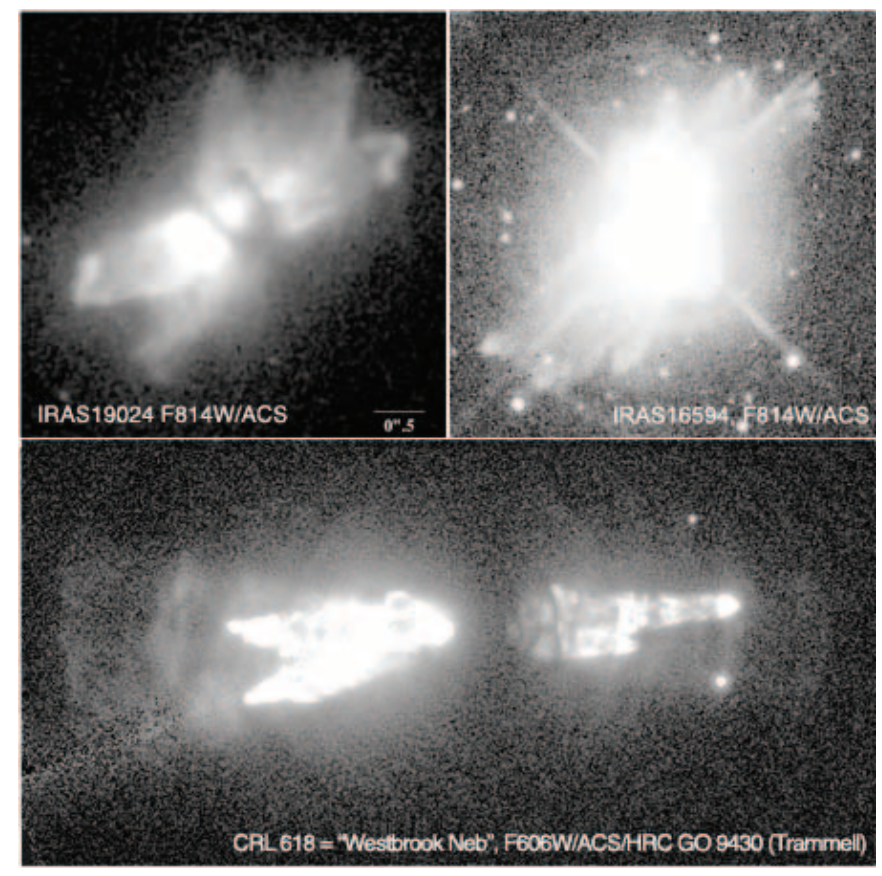

FIG. 1.-Examples of multipolar structure observed with the Hubble Space Telescope in several proto-planetary nebulae. Note the V-shaped heads of the thin fingerlike extensions (see text for discussion).

star. The simulations showed that long, thin morphologies similar to jets were readily obtained along with multiple rings associated with vortex shedding and the breakup of the clump. The authors suggested that such "impulsive" models may be useful in PPNs as well. Such a scenario is very different from the jet-driven explanation for PPNs/PNs. In this paper we seek to explore the usefulness of the clump picture.

Soker (2000) has analytically explored the role of jets in PNs. In the excellent study of Lee \& Sahai (2003), simulations of jets as the drivers of PPNs were presented, including detailed comparisons with observations. Our goals in this paper are more modest. In what follows we take a first step in the exploration of the clumps versus jets issue by examining two-dimensional (2D) and three-dimensional (3D) pairs of simulations, with each pair consisting of either a steady jet impinging on a circumstellar gaseous medium or a clump of gas which is fired ballistically through the same medium along a trajectory corresponding to the direction of flow in the steady jet. ${ }^{4}$ Both the jet and the clump are assumed to be magnetically launched, although no attempt to model the launch mechanism is made here, and the simulations are purely hydrodynamic. For the present, we are merely interested in examining how the clump and jet differ in their effect on the surrounding circumstellar medium. As we will show, the jet and clump models show differences which require further study, but the clumps provide at least as good (or better) an account of key observational characteristics. Given the fact that in some cases multiple outflows are seen in a single object (such as CRL 618), the clump model may be more plausible since what are often interpreted as multiple "jets" could instead arise naturally from the fragmentation of an explosively driven polar directed shell. The "clumps" arising from this fragmentation would not be constrained to flow along the axis of rotation of the central object, as in the case of the jets discussed above, and would account

\footnotetext{
${ }^{4}$ In a related study Raga et al. (2007) have also recently presented a model of the "three-dimensional structure of a radiative, cosmic bullet flow."
}

TABLE 1

Simulation Parameters

\begin{tabular}{|c|c|c|}
\hline Parameter & Value (2.5D) & Value $(3 \mathrm{D})$ \\
\hline \multicolumn{3}{|l|}{ Jet Model } \\
\hline Radius, $r_{j}(\mathrm{AU})$ & 500 & 500 \\
\hline Computational cells per $r_{j} \ldots \ldots \ldots \ldots \ldots \ldots \ldots \ldots$ & 48 & 24 \\
\hline 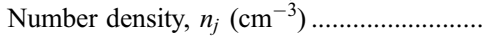 & 500 & 500 \\
\hline 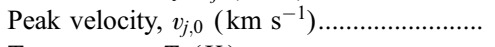 & 100 & 100 \\
\hline 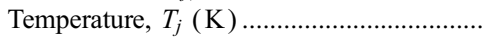 & 200 & 200 \\
\hline Nominal ambient density, $n_{a}\left(\mathrm{~cm}^{-3}\right) \ldots \ldots \ldots$ & 500 & 500 \\
\hline Ambient temperature, $T_{a}(\mathrm{~K}) \ldots \ldots \ldots \ldots \ldots \ldots \ldots \ldots \ldots \ldots$ & 200 & 200 \\
\hline 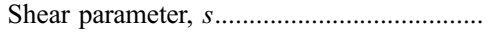 & 0.9 & 0.9 \\
\hline \multicolumn{3}{|c|}{ Clump Model } \\
\hline 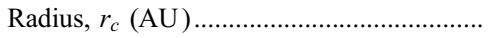 & 500 & 500 \\
\hline 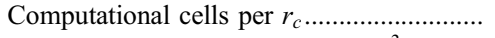 & 48 & 24 \\
\hline Nominal number density, $n_{0}\left(\mathrm{~cm}^{-3}\right) \ldots \ldots \ldots . .$. & 500 & 500 \\
\hline 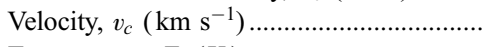 & 100 & 100 \\
\hline 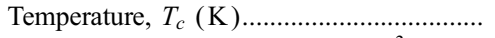 & 200 & 200 \\
\hline Nominal ambient density, $n_{a}\left(\mathrm{~cm}^{-3}\right) \ldots \ldots \ldots$ & 500 & 500 \\
\hline Ambient temperature, $T_{a}(\mathrm{~K}) \ldots \ldots \ldots \ldots \ldots \ldots \ldots \ldots \ldots \ldots$ & 200 & 200 \\
\hline
\end{tabular}

naturally for the creation of multiple outflows of similar or equal age driven in slightly different directions.

We note also that new models of binary stars in the context of PNs (Nordhaus \& Blackman 2006; Nordhaus et al. 2007) show the extent to which envelope ejection can be shaped by gravitational interactions. In Nordhaus \& Blackman (2006), common envelope scenarios which lead to aspherical mass loss (including disk creation and possible MHD launching) were articulated. In Nordhaus et al. (2007) common envelope models were explored as the source of differential rotation in the primary that could drive strong dynamo supported magnetic fields. These models showed that while single stars may, in some cases, be able to support a strong field over AGB timescales, binary interactions were highly effective at creating the fields needed to power PPN outflows at the evolutionary moment when they will be required. As we will see, such models provide strong theoretical support for the scenario we argue for in this paper.

In $\S 2$ we provide information concerning the numerical methods used, details of the jet and clump models, and a discussion of the initial conditions. In $\S 3$ we discuss the results of our simulations, and in $\S 4$ we summarize our conclusions.

\section{COMPUTATIONAL METHODS AND INITIAL CONDITIONS}

We have carried out two pairs of hydrodynamic simulations (one "medium resolution" 3D pair and one "high resolution" 2.5D pair). Each pair consists of a jet and clump, respectively, with each parameterized to be as similar to one another as possible. Specific parameter values for the jet, clump, and ambient medium for each simulation are given in Table 1 . The simulations are performed using the AstroBEAR code, which is an extension of the BEARCLAW adaptive mesh refinement (AMR) package for solving conservation laws (for a detailed description of the AstroBEAR package see $\S 3$ of Cunningham et al. 2006). The domain is a rectangular box with a square cross section and with the $x$-axis chosen to intersect the center of the left square face of the domain. The origin of the coordinates is placed at this point of intersection. The clump and jet are launched along the $x$-axis and placed so that their centers coincide with it. The jet 
was modeled in 3D with a circular cross section of radius $r_{j}$ (in $2.5 \mathrm{D}$ the jet cross section reduces to a line segment of length $2 r_{j}$ and a thickness of one computational cell). The jet was launched into the domain from a set of fixed cells along the domain boundary. To prevent the expansion of the jet inflow boundary with time, a ring of zero velocity and with outer radius $1.125 r_{0}$ was maintained around the jet-launching region. The velocity profile of the jet was smoothed about a nominal value $v_{j, 0}$ according to

$$
v_{j}=v_{j, 0}\left[1-(1-s)\left(\frac{r}{r_{0}}\right)^{2}\right],
$$

where $s$ is a shearing parameter taking values between 0 and 1 and for the jet simulation presented here is set equal to $s=0.9$. The clump was modeled in 3D as a spherical overdensity of radius $r_{c}=r_{j}$. (The sphere reduces to a circle in 2.5D.) Its initial position in the domain is chosen so that its center is located at the point

$$
\boldsymbol{r}_{c, 0}(x, y, z)=\left(2 r_{c}, 0,0\right)
$$

and the density of the clump as a function of location within the clump is

$$
n_{c}(r)=n_{a}(r)+n_{0}\left[1-\left(\frac{\left|\boldsymbol{r}-\boldsymbol{r}_{c, 0}\right|}{r_{0}}\right)^{2}\right]
$$

where

$$
n_{a}(r)=\min \left(n_{0}, \frac{n_{0} r_{0}^{2}}{r^{2}}\right)
$$

is the ambient number density profile in regions of the domain unoccupied by jet or clump gas, and where $r^{2}=x^{2}+y^{2}+z^{2}$, $n_{0}$ is the nominal ambient number density, and $r_{0}$ is a characteristic length taken to be equal to the jet or clump radius. The $3 \mathrm{D}(2.5 \mathrm{D})$ simulations are carried out on a base grid with a resolution of 6 (12) cells per jet/clump radius and with two levels of AMR refinement providing an effective resolution of 24 (48) cells per jet/clump radius. In all cases, radiative cooling is modeled using the atomic line cooling function of Delgarno \& McCray (1972), and we do not attempt to follow the detailed ionization dynamics or chemistry of the cooling gas. Given that both models give rise to similarly expanding shells of shock-heated gas, we do not expect this simplification to materially affect our conclusions.

We note that our choices of initial conditions were determined in part by computational efficiency. Strong cooling in the code requires adequate resolution of cooling layers behind shocks. Even with AMR such resolution can be prohibitively expensive in terms of computational time. What matters for the dynamics, however, is not the scaled value of the parameters but the dimensionless numbers associated with the flow. In this case it is the socalled cooling parameter $\chi=d_{\text {cool }} / d_{\text {hydro }}$ which determines the dynamics (Blondin et al. 1990). Here $d_{\text {cool }}$ is an appropriately chosen cooling length scale, and $d_{\text {hydro }}$ is a corresponding hydrodynamic timescale. If $\chi>1$, then the flow is in the adiabatic regime, and $\chi<1$ delimits the radiative regime with thin cooling layers behind the shock. In the Appendix we demonstrate that all of our simulations are well within the radiative regime throughout their evolution except in a few cases at the very end. The shock cooling layers remain well resolved, allowing us to adequately capture their dynamics. Thus, while it is likely that the densities of the circumstellar environments of real PPNs are much greater
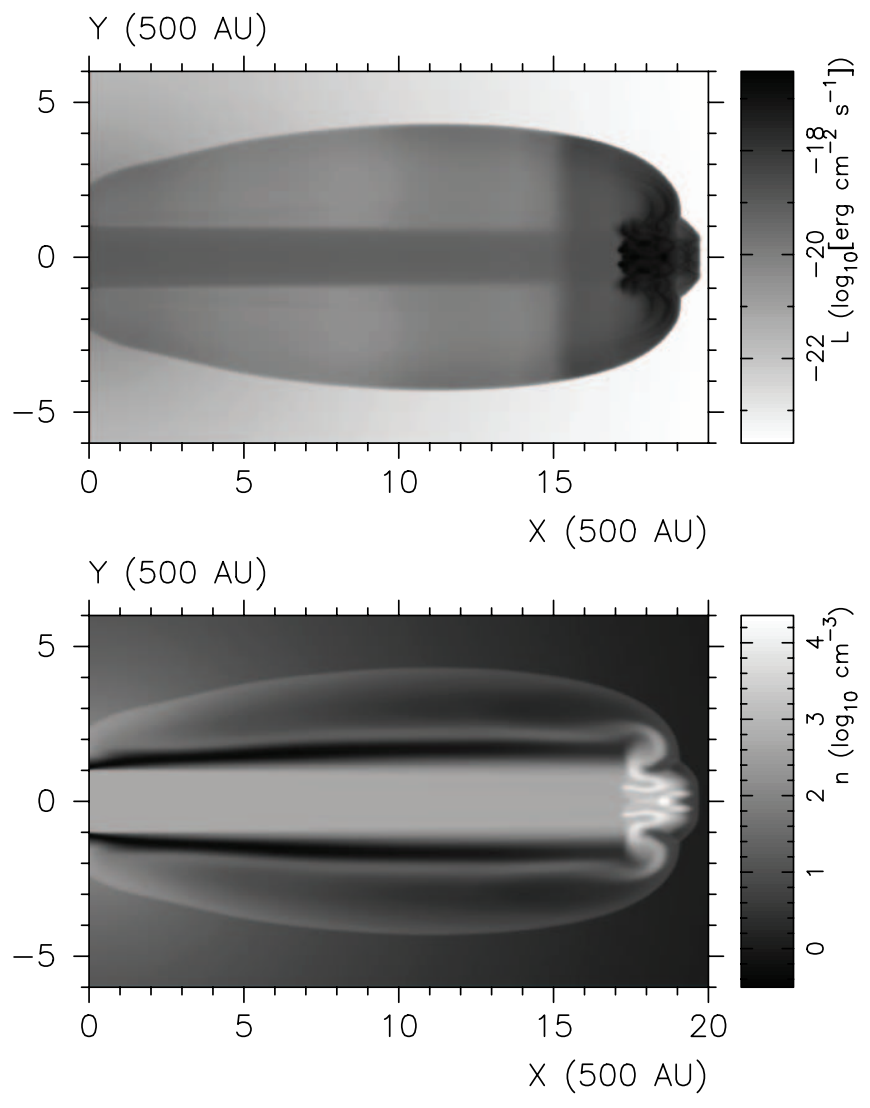

FIG. 2.- Three-dimensional jet at time $t \simeq 636 \mathrm{yr}$. Top, integrated emission assuming atomic line cooling; bottom, base-10 logarithm of density.

than the choices made here, we can be assured that once in the radiative regime, decreasing $\chi$ does not change the global behavior of the flows, and only the details of small-scale instabilities are modified.

\section{RESULTS: MORPHOLOGY AND KINEMATICS}

Results of our simulations are presented in Figures 2-7. In Figures 2 and 3 we present the results of medium-resolution ( 24 cells per radius), 3D simulations of one jet and one clump, respectively. The length of the domain in these simulations is 20 computational units, with one computational unit corresponding to a physical scale of $500 \mathrm{AU}$. (One computational unit is also the value chosen for the half-width of the jet and clump.) In each figure the upper image shows a plot of emission integrated along the line of sight, which in the case of these figures is perpendicular to the plane of the image. The lower image in each figure is a plot of the logarithm of density in a plane coincident with the $x-y$ plane. The images show the jet and clump near the end of their respective runs at time $t \simeq 498 \mathrm{yr}$ for the case of the clump and at time $t \simeq 636 \mathrm{yr}$ for the case of the jet. The resolution is seen to be sufficient to capture vortex-shedding events in both simulations. It is also evident from these images that while one can easily distinguish jet from clump in the density maps, the emission maps are quite similar. We note that the clump gives rise to a somewhat more collimated flow, while the jet bow shock expands laterally at a greater rate than the clump bow shock. The jet, which has the same initial width as the clump, also lags behind the clump in its forward motion. This is likely due to the streamlining that occurs as the head of the clump is reduced in size as material is ablated away via its interaction with the ambient medium. 


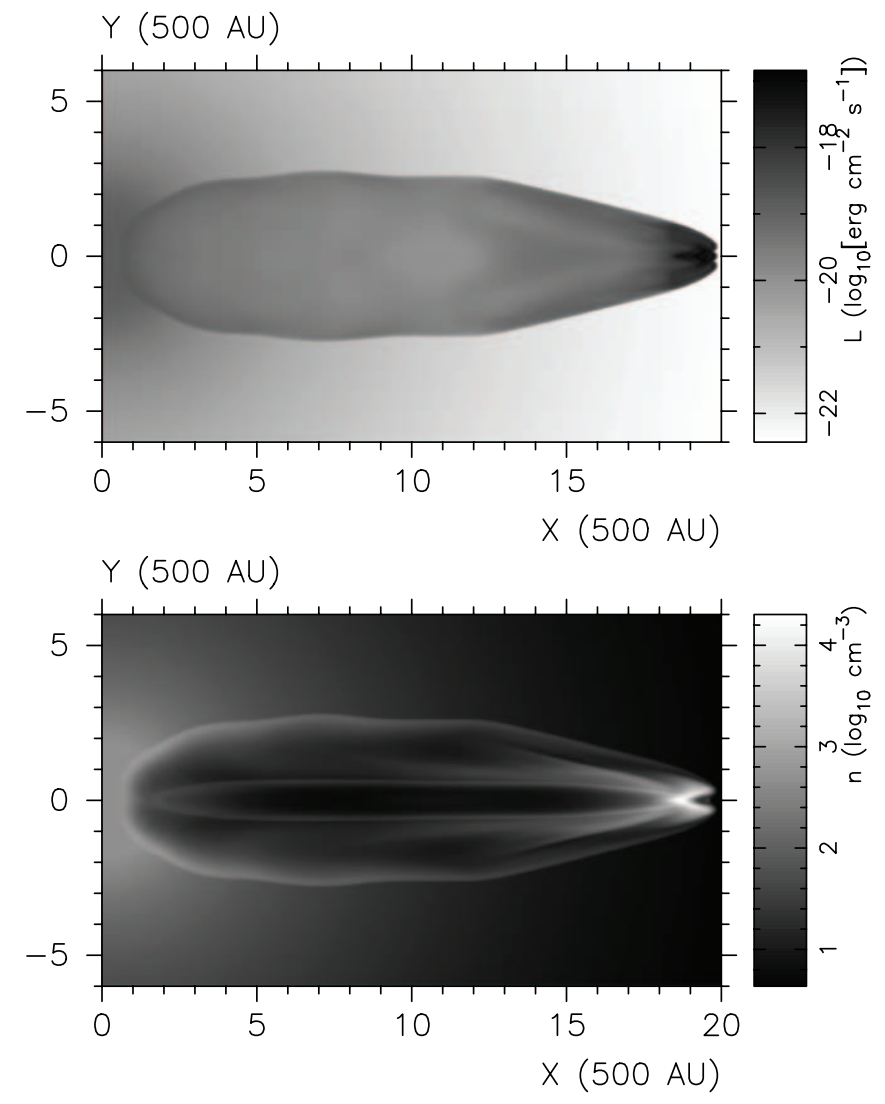

FIG. 3.-Three-dimensional clump at time $t \simeq 498$ yr. Top, integrated emission assuming atomic line cooling; bottom, base-10 logarithm of density.

Due to limits on computational resources, it was necessary to impose limits on the resolution and run time of the 3D simulations from which the images in Figures 2 and 3 are taken. The simulations end just as the vortex-shedding events begin to have an interesting effect on the nebular environment. To explore this stage further we carried out the second pair of 2.5D simulations mentioned above. In these high-resolution simulations, the effective resolution was doubled to 48 cells per jet/clump radius, the length of the domain was doubled, and the transverse dimensions of the domain were enlarged in an attempt to accommodate the lateral expansion of the jet/clump bow shock (this latter adjustment was successful only for the case of the clump). Results from these simulations are presented in Figures 4-7. In Figures 4 and 5 we again present images of the logarithm of density for the jet and clump, respectively - this time reflected about the axis of symmetry. Both figures show the simulations at various stages of the flow. We observe that the differences found between the two models in the 3D simulations - i.e., the faster domain crossing time and the higher degree of collimation exhibited by the clumpare seen again in these images. The vortex shedding, however, is now captured with greater clarity for both jet and clump, and we begin to see significant qualitative differences in the manner in which these events unfold. In particular we note that shedding events are much more frequent in the case of the clump. These results mirror those found by Poludnenko et al. (2004). It is noteworthy that their study used a different integration scheme from that used here. AstroBEAR has a number of schemes built into it, and in the Poludnenko study a wave-propagation scheme was used (LeVeque 1997), while here a MUSCL-Hancock method is used. The fact that the basic morphology of clumps driving bow shocks dominated by vortex-shedding events is recovered

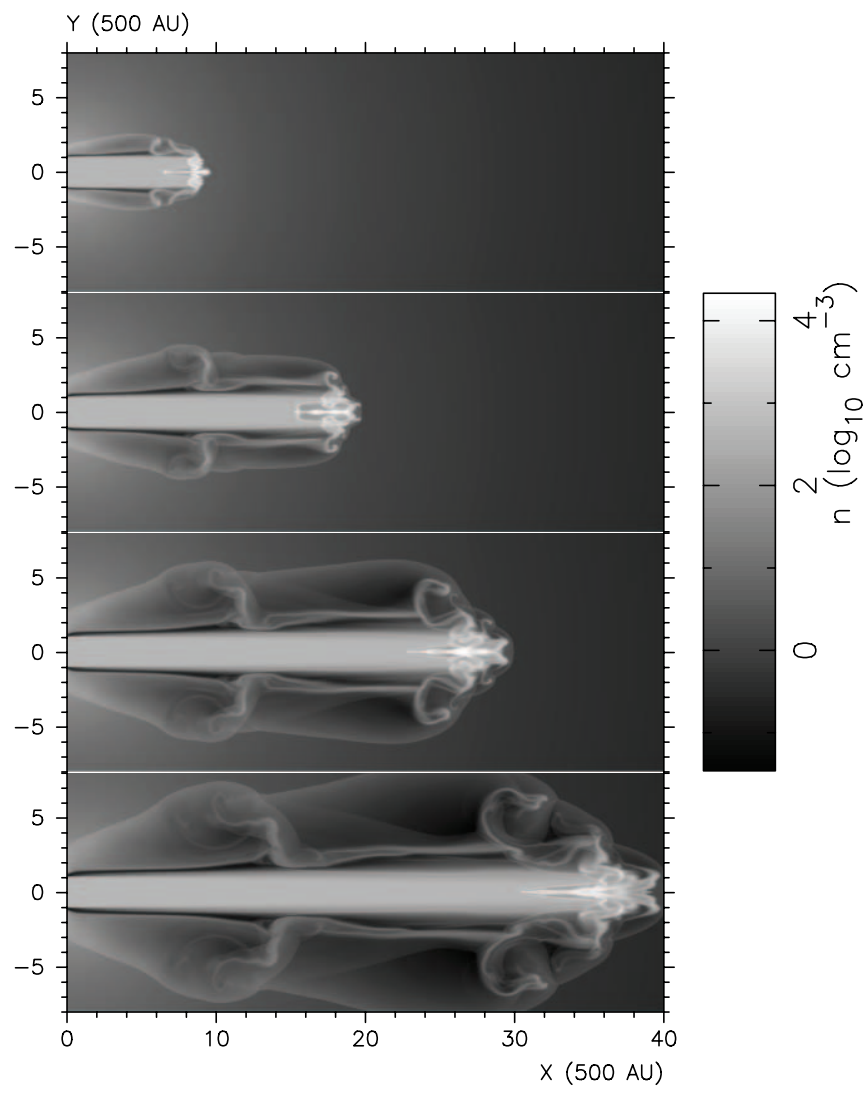

FIG. 4.-Density of $2.5 \mathrm{D}$ jet at times $t \simeq 336,628,896$, and $1165 \mathrm{yr}$ ( from top to bottom). The data are shown here reflected about the axis of symmetry.

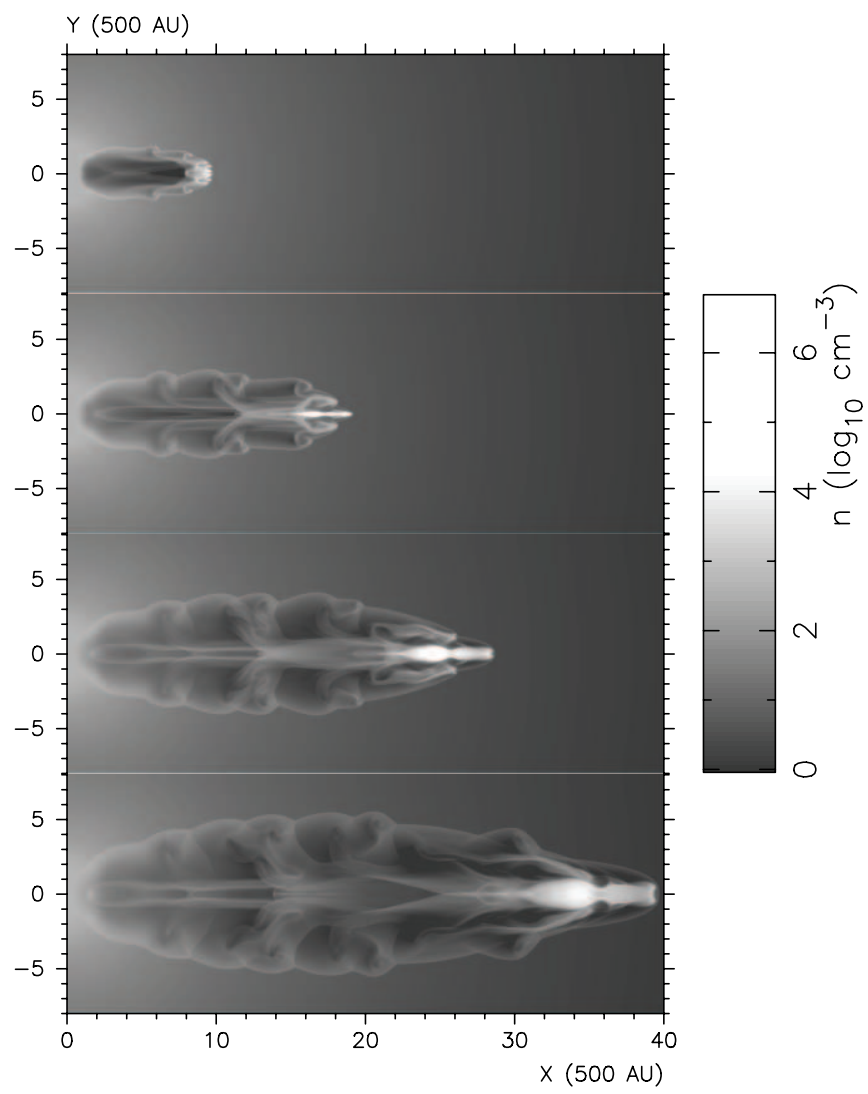

FIG. 5.-Density crosscut of 2.5D clump at times $t \simeq 208,477,753$, and $1082 \mathrm{yr}$ ( from top to bottom). The data are shown here reflected about the axis of symmetry. 

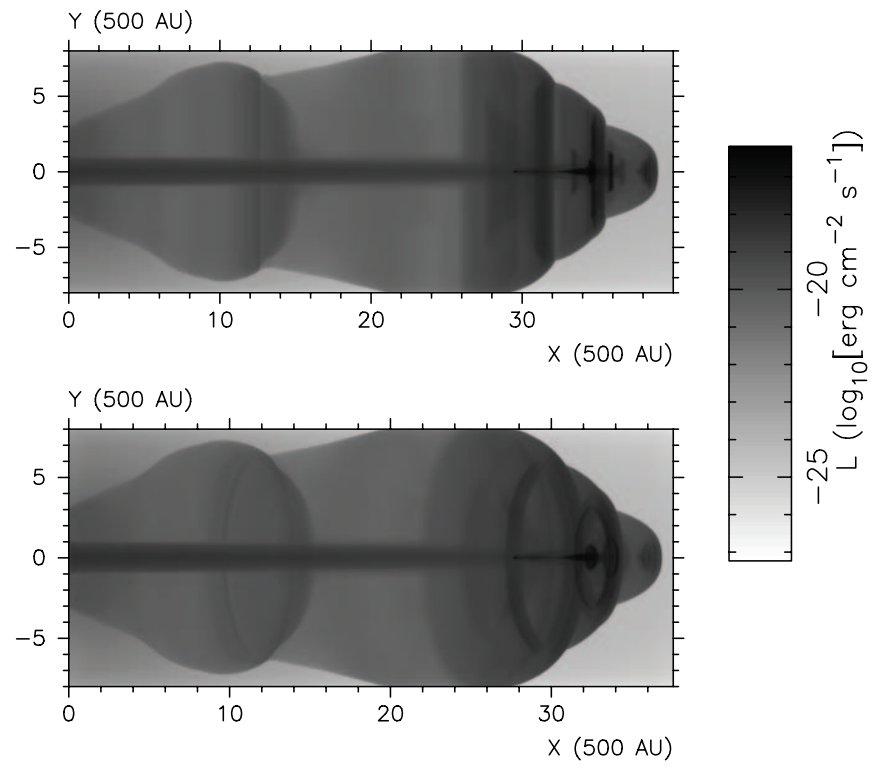

FIG. 6.- Integrated emission of $2.5 \mathrm{D}$ jet at time $t \simeq 1132 \mathrm{yr}$. The data are shown here rotated about the axis of symmetry and with angles of inclination of the symmetry axis with respect to the image plain of $\theta=0^{\circ}$ (top) and $\theta=20^{\circ}$ (bottom).

using both schemes gives us confidence in this aspect of the dynamics.

\subsection{Morphology}

To get a better sense of how the differences between the models might appear observationally, we present integrated emission maps for the jet and clump, respectively, in Figures 6 and 7. These figures were produced by calculating the effect on the lineof-sight emission resulting from a rotation of the cylindrically symmetric data set about the axis of symmetry. The intensity shown, which does not distinguish among cooling lines, was determined according to

$$
I_{i, j, k}=\Sigma_{k} n_{i, j, k}^{2} \Lambda\left(T_{i, j, k}\right),
$$

where $i, j$, and $k$ refer to the $x$-, $y$ - and $z$-directions in the final data cube created by rotating $n(r, z)$ and $T(r, z)$ about the axis of symmetry, and $\Lambda$ is the cooling function.

It is worth mentioning that there is evidence suggesting that much of the radiative cooling occurring in PPNs is due principally to atomic cooling lines in the far-infrared (FIR) associated with photodissociation regions (PDRs) rather than shocks (Fong et al. 2001). However, the source of this radiation is the undisturbed medium beyond the regions of flow under consideration here. Instead we focus on the shock-heated gas associated with the jet and clump disturbances. Ample evidence exists indicating the presence of such gas in PPNs (e.g., CRL 618; Trammel \& Goodrich 2002; Trammel et al. 1993; Fong et al. 2001).

Figures 6 and 7 correspond to the final frames in each of Figures 4 and 5, respectively. Each figure provides two views of the data: one in which the angle of inclination of the symmetry axis with respect to the image plane, $\theta$, is $0^{\circ}$; and one in which it is $20^{\circ}$. One difference between the jet and clump cases appears in the shape of the head of the bow shock. A clump has a finite reservoir of mass which interacts with the ambient medium. As the clump propagates down the grid, it drives a (bow) shock wave into the ambient medium. A second shock passes through the clump, heating and compressing it. When cooling is present this
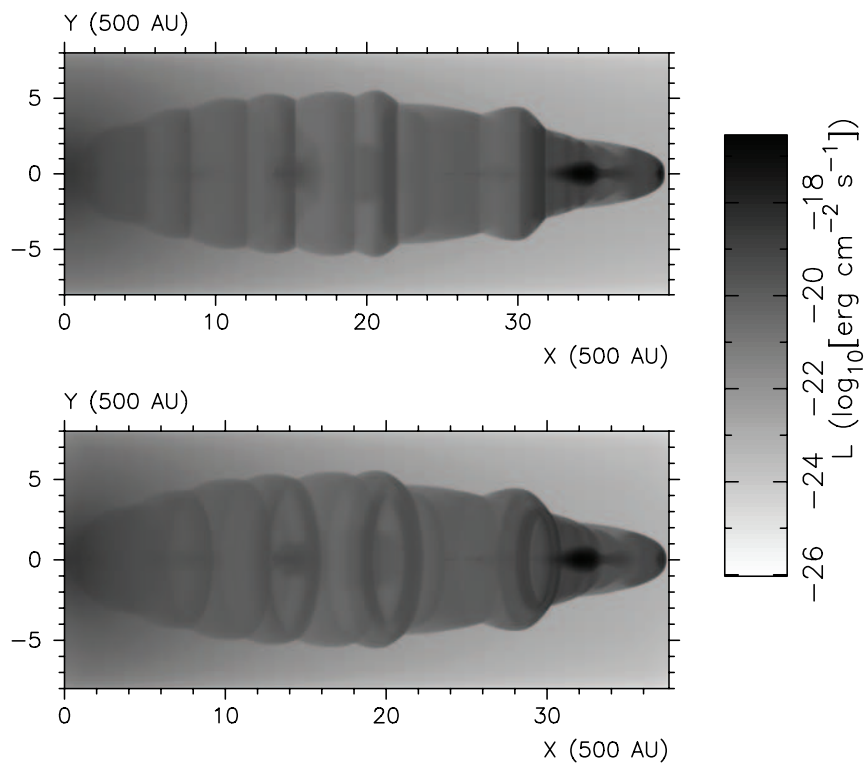

FIG. 7.- Integrated emission of 2.5D clump at time $t \simeq 1082 \mathrm{yr}$. The data are shown here rotated about the axis of symmetry and with angles of inclination of the symmetry axis with respect to the image plain of $\theta=0^{\circ}$ (top) and $\theta=20^{\circ}$ (bottom).

"transmitted shock" first leaves the clump flattened. As material is then ablated away via the interactions with the ambient medium the remaining clump material becomes dense and streamlined in the direction of propagation. At later times in the simulation the dense core of the clump drives a V-shaped bow shock head. In the case of a jet the situation is different. The jet head drives a bow shock into the ambient medium and a transmitted shock, called a "jet shock," propagates back into the jet material. Decelerated jet material flows transverse to these shocks inflating a cocoon behind the wings of the bow shock. Unlike the clump, however, there is always more high-speed material behind the jet shock/ bow shock pair to resupply the interaction. Thus, with material continuously flowing into the cocoon, the bow shock head remains wider and takes on a flatter, more $U$-shaped configuration. Such a distinction between V-and U-shaped flows may be important in comparing with observations. We note that both the $2.5 \mathrm{D}$ and $3 \mathrm{D}$ simulations show this difference. The characteristic length scale of these configurations can be seen from the figures to be $\sim 1500 \mathrm{AU}$. Supposing a typical distance to the source to be $\sim 1 \mathrm{kpc}$, this corresponds to an angular width of $\sim 3^{\prime \prime}$ suggesting that such shapes are resolvable with present-day instruments. We note, however, that the axial symmetry of our $2.5 \mathrm{D}$ simulations tends to enhance features on the axis, and that our 3D runs do not yet have the resolution to accurately track the breakup of the clump. Thus, the $\mathrm{V}$ - and $\mathrm{U}$-shaped bow shock head distinction still merits further study at higher resolution.

Vortex shedding provides another morphological distinction. In the case of the clump, the relatively frequent shedding events have led to a series of thin, irregularly spaced rings of enhanced intensity centered on the symmetry axis. These are reminiscent of the ringlike structures observed in some collimated PPN outflows (see, e.g., Fig. 1 and Trammell \& Goodrich 2002). The shedding events occurring in the jet simulation lead to similar structures, but these are less frequent and somewhat more bandlike in character. The qualitative differences in the manner in which these rings form in the outflows depending on whether one models them as jets or clumps might suggest a means of distinguishing between the two models in observations. One must, once again, 

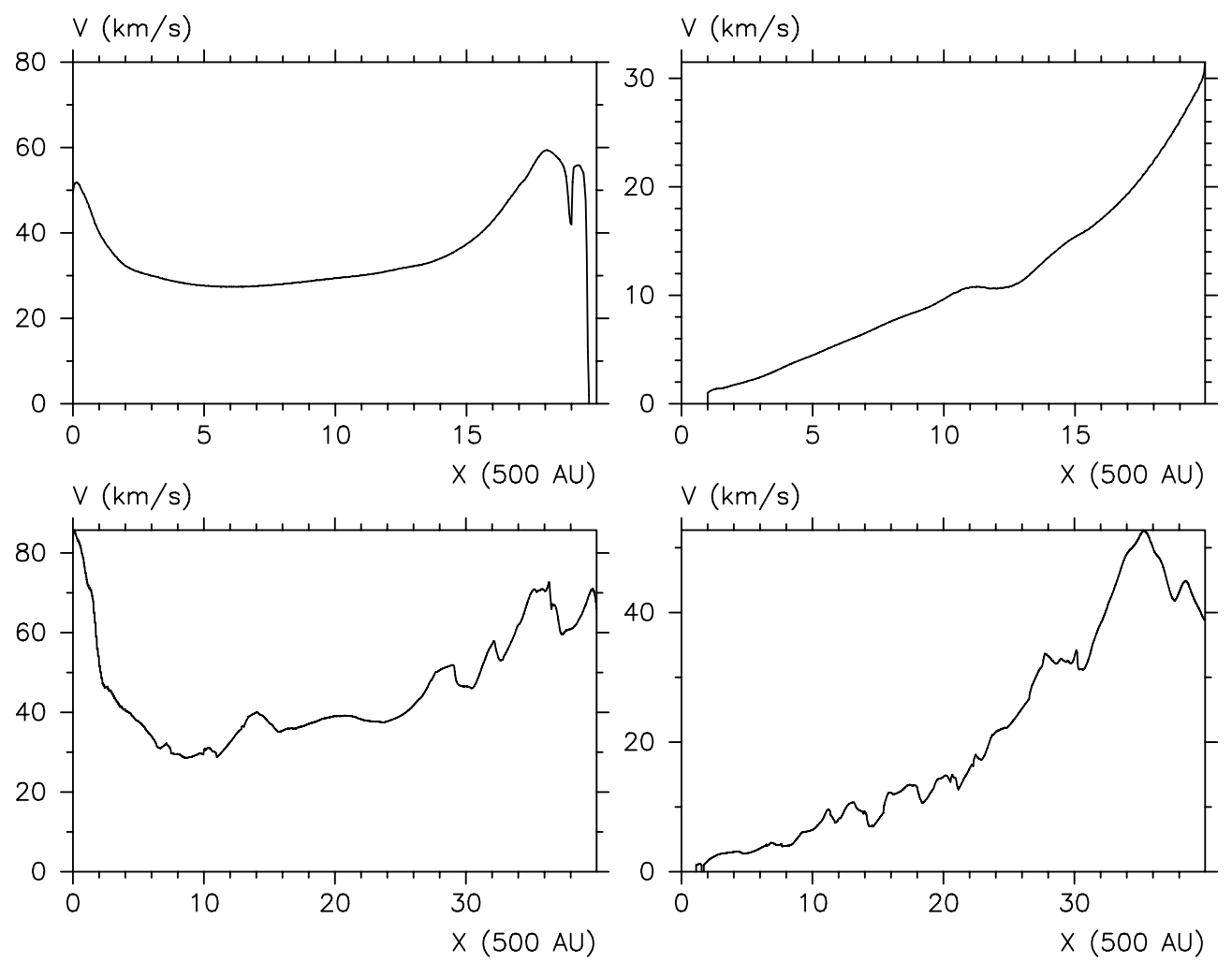

FIG. 8.-Comparisons of weighted average parallel flow velocity, $\left\langle v_{x}\right\rangle$, vs. distance from the flow origin for the $3 \mathrm{D}$ simulations $($ top row $)$ calculated at time $t \simeq 636$ yr,

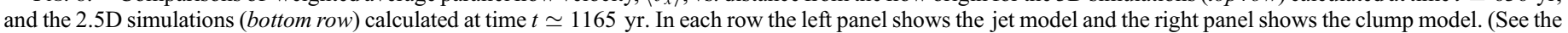
text for an explanation of the weighting.)

be careful not to overinterpret these results due to limits on the resolution and the fact that these simulations are $2.5 \mathrm{D}$. We thus conclude that the high-resolution 2.5D simulations lend weight to the assertion that clumps and/or jets can account for observed ringed structures, but neither can be ruled out as a model for the collimated outflows observed in the environments of PPNs. In the meantime, we note that this conclusion in itself is important with respect to PPN studies as we will discuss in $\S 4$.

It is also noteworthy that Lee \& Sahai (2003) attempted to model the rings via a pulsed jet. Each ring became associated with an "internal working surface" where faster moving material swept over slower moving material. The internal shocks lead to transverse motions of shocked material which impinge on the bow shock. As might be expected, the strength of the emission from these shocks decreased as the pulse traveled down the length of the beam. Such dimming of the rings with distance from the source is not what is observed in CRL 618. The clump on the other hand produces the opposite kind of pattern, as ablation events on the clump lead to rings that are brightest and densest closer to the head of the bow shock.

Finally we note that for both models, the temperatures of the shocked gas were found to be $\sim 10^{5} \mathrm{~K}$, corresponding to peak values of the cooling function $\Lambda$, placing these simulations firmly in the radiative regime in spite of the relatively small values of density chosen for the models. We may therefore be confident that the morphological features evident in the figures presented here are qualitatively substantially the same as what one may expect when considering values of density matching more closely those found in real systems (see Appendix).

\subsection{Kinematics}

In addition to comparisons of morphology, it is also important to consider the flow kinematics, since observed flows are often seen to exhibit "Hubble flow" characteristics - that is, the flow velocity is observed to increase linearly with respect to distance from the flow origin. To address this issue we present in Figure 8 plots of the $x$-component of the flow velocity averaged over the directions transverse to the flow $\left\langle v_{x}\right\rangle$. Velocity tracers were not used in our simulations. Therefore, in order to differentiate between mildly perturbed ambient gas and gas that is fully involved in the flow, values of velocity $\lesssim 0.01 v_{0}$ were ignored, where $v_{0}$ is the initial velocity of the clump or jet gas. The top row of the figure shows, from left to right, respectively, the results for the $3 \mathrm{D}$ jet and clump, while the bottom figure shows the results for the $2.5 \mathrm{D}$ jet and clump. The data for these plots are taken from times near the end of the simulation when the flows have crossed most of the domain. In the two plots involving clumps (right), there are large regions of the flow for which the variation of velocity with distance is roughly linear. The jets (left), on the other hand, fail to model this behavior altogether. Comparison of the $3 \mathrm{D}$ clump plot (top right) to the $2.5 \mathrm{D}$ case yields an interesting result. Recall that the first vortex-shedding events in both clump and jet were observed to occur in the 3D simulations shortly before the end of the simulation. Because of this they do not have time to perturb the flow in a way that might be noticeable in these plots. However, when we examine this phenomenon kinematically in the extended spatial domain allowed by the $2.5 \mathrm{D}$ simulation, we find that while the vortex-shedding events appear to perturb the kinematics of both jet and clump, these perturbations do not alter the overall qualitative character of the flows in either case.

To further examine the kinematics of our simulated flows, we have also produced a set of synthetic position-velocity (PV) diagrams for the 2.5D simulations. These are presented in Figure 9. Once again, as in Figures 6 and 7, we present our results in pairs corresponding to values of $0^{\circ}$ and $20^{\circ}$ for the angle of inclination, 

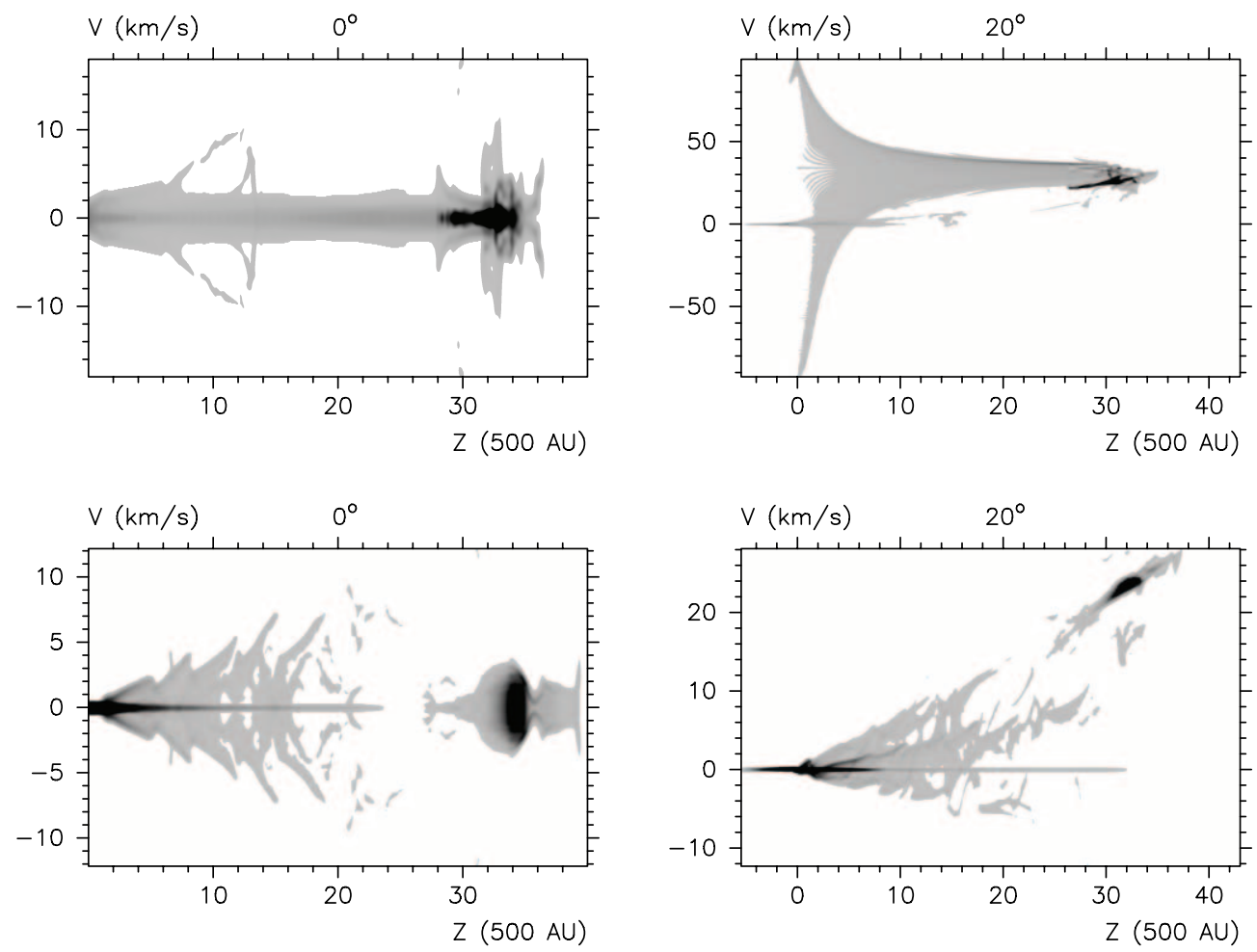

FIG. 9. - Position-velocity diagrams at time $t \simeq 1082 \mathrm{yr}$ for the $2.5 \mathrm{D}$ jet (top row) and the $2.5 \mathrm{D}$ clump (bottom row) assuming inclination angles of $\theta=0^{\circ}$ (left) and $\theta=20^{\circ}$ (right).

$\theta$, of the flow symmetry axis with respect to the image plane. These plots were produced by calculating the velocity structure along the line of sight and with the "slit" placement taken to be along the projected axis of symmetry. Results for the jet are given in the top row of the figure, and results of the clump are given in the bottom row. In these images, the differences between the clump and jet are even more striking. For either angle of inclination, the velocity structure of the jet cannot be said to be even approximately Hubble-like. The clump however, continues to exhibit line-of-sight velocity structure indicative of a linear increase with distance along the projected direction of flow. The effect is particularly apparent in the case of the flow that is inclined with respect to the image plane. These results, and those of Figure 8, suggest that it may be possible to distinguish between outflows from steady jets and those from explosive events through a careful examination of their kinematics.

One caveat which must be considered in these results is the role of emission. Because our models do not track emission from individual species, we cannot separate the emission at the bow shock from that within the jet or from the shocked jet material. In Ostriker et al. (2001), a model for the emission from a jet-driven bow shock was presented which showed a characteristic spur pattern in synthetic PV diagrams. The spur exhibits a rapid drop in velocity away from the tip of the bow shock. Lee \& Sahai (2003) found a range of patterns in their jet simulations which in some cases took on the spur morphology. Thus, our results are suggestive of the differences between jets and clumps and indicate that clumps appear to be better, in general, at recovering quasilinear increases in velocity along the nebular outflow lobe.

\subsection{Kinematic Models}

In order to interpret our results we consider the time-dependent distortion of the clump gas during the evolution of the outflow.
Strongly radiative, hypersonic clouds of any geometry will be rapidly compressed into a thin ballistic sheet after ejection by the outflow progenitor. We therefore consider the motion of a cylindrically symmetric disk with surface density $\zeta(r)$ and velocity $v(r, t)$, where $v(r, 0)=v_{0}$, to model the time-dependent evolution of the clump gas. The equation of motion for a differential ring of the disk under the ram pressure of the ambient gas of density $\rho$ is given by

$$
\rho v^{2}(r, t)=-\zeta \frac{d v}{d t}
$$

Because the outflow bow shock is convex, most of the outflowentrained ambient gas will be swept outside the path of the clump into the bow shock. We therefore neglect accretion of ambient material onto the clump and the kinematics of ambient material ejection in this model. Because the disk is hypersonic in a strongly cooling environment, we consider the model disk to be ballistic, neglecting pressure forces. For simplicity we also take the density of the ambient gas to be constant. Thus, the equation of motion for a differential ring of clump gas with radius $r$ integrates to

$$
v=\frac{v_{0}}{1+\rho v_{0} t / \zeta} .
$$

The distance traversed by the ring is given as

$$
L(\zeta, t)=\int_{0}^{t} v(t) d t=\frac{\zeta}{\rho} \ln \left(1+\frac{\rho v_{0} t}{\zeta}\right) .
$$

The quantities $v, t$, and $L$ all refer to the same ring. What differentiates one ring of material from another is the parameter $r$. 
Now at some late time $t$, we imagine the rings to have been distributed over the length of the outflow with this distribution depending on $r$. For this fixed value of $t$, we are interested in plotting the velocity of each ring against its corresponding distance. The $r$-dependence of $v$ and $L$ enters into the expressions for these quantities through the surface density $\zeta$. We therefore model $\zeta$ by assuming that the clump of gas from which our disk formed was initially spherical, of constant volume mass density $\sigma$, and compressed in such a way that all material within the volume of the clump and lying along a given line passing through the clump in the direction of its motion remains on this line after compression. Then

$$
\zeta(r)=2 \sigma r_{0} \sqrt{1-\left(r / r_{0}\right)^{2}}
$$

where $r_{0}$ is the radius the clump/disk. We introduce the following dimensionless quantities:

$$
\begin{gathered}
\tilde{r}=\frac{r}{r_{0}}, \\
\tilde{v}=\frac{v}{v_{0}}, \\
\tilde{t}=\frac{\rho v_{0} t}{2 \sigma r_{0}}, \\
\tilde{L}=\frac{\rho}{2 \sigma r_{0}} L .
\end{gathered}
$$

Our parametric equations are

$$
\begin{gathered}
\tilde{v}=\left(1+\frac{\tilde{t}}{\sqrt{1-\tilde{r}^{2}}}\right)^{-1}, \\
\tilde{L}=\sqrt{1-\tilde{r}^{2}} \ln \left(1+\frac{\tilde{t}}{\sqrt{1-\tilde{r}^{2}}}\right) .
\end{gathered}
$$

The value of $\tilde{t}$ is chosen by assuming $\sigma \gtrsim 2 \rho$, and by noting that at late times $v_{0} t \gtrsim r_{0}$. In our 2.5D simulations we have $v_{0} t / r_{0} \sim$ $4 \tilde{t} \simeq 20$ making $\tilde{t} \simeq 5$. Figure 10 shows a plot of $v$ versus $L$ in astronomically relevant units with this choice of $\tilde{t}$. For purposes of comparison, a line with an appropriately chosen slope and intercept is plotted as well. In spite of the simplicity of our analytical model, a comparison of this plot with the top right plot of Figure 8 reveals good agreement between the two. Both curves exhibit a small concave curvature for small $L$ while becoming increasingly linear with increasing $L$ (the downward turn in the 2.5D-simulation-based plot in Fig. 8 [bottom right] results from extending the plot into regions not yet reached by the clump). We also find that the range of $L$-values over which the curve shows linear behavior increases for increasing $\tilde{t}$, implying a correlation between the kinematical ages of PPN outflows and the extent to which they are observed to exhibit Hubble flow. We take this calculation as further evidence that our simulations are accurately capturing the dynamics of the flow and the relevance of bullet models for PPNs.

\section{DISCUSSION AND CONCLUSIONS}

In this paper we have examined the results of two pairs of simulations intended to model the gross morphological and kinematical properties of PPNs. Our primary purpose was to ask if clump models could perform as well as jet models at recovering these

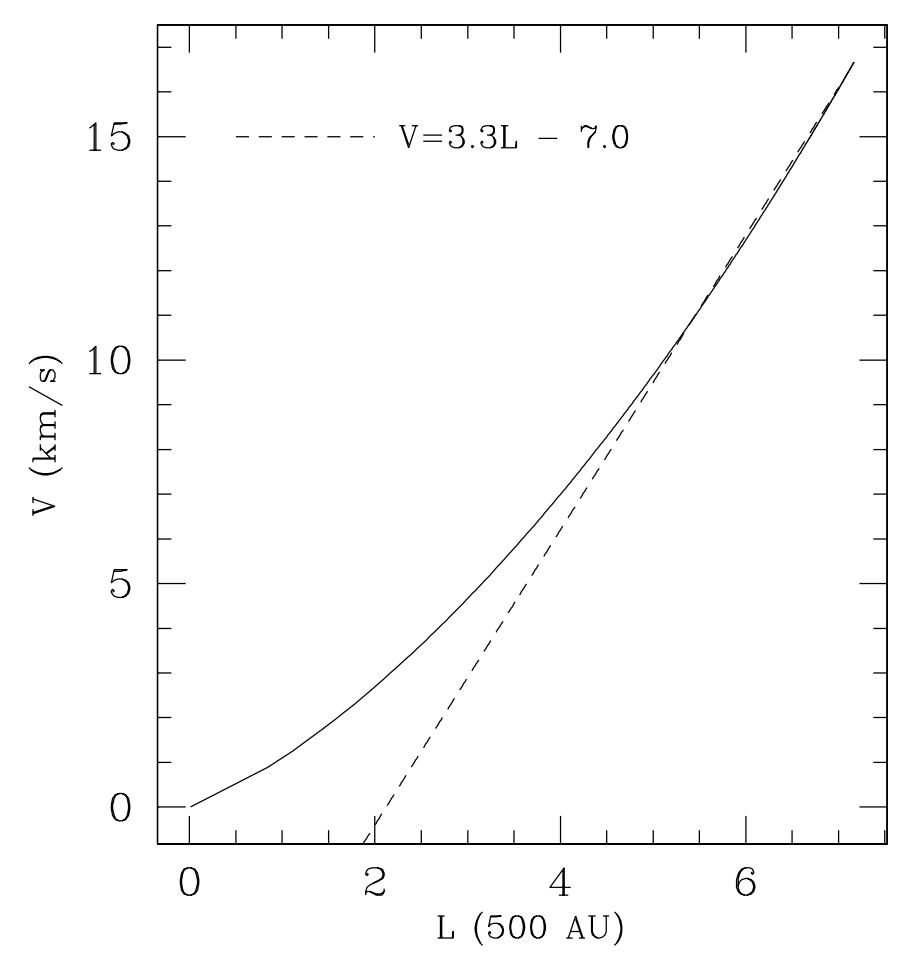

FIG. 10.-Plot of $V(r)$ vs. $L(r)$ for $\tilde{t}=5$. See $\S 3.3$ for an explanation of this figure.

properties. As was discussed in $\S 1$, MHD models of PN shaping have been explored by a variety of authors and in a variety of forms. These models, particularly the magnetocentrifugal launch and collimation scenarios, are what motivate this paper. Below we elaborate on these models and explain their connection with the jet/clump comparisons presented in this paper, and then present the conclusion of our study.

Two distinct classes of model for the magnetic shaping of winds in PNs and PPNs have been suggested to date. First there is the magnetized wind bubble (MWB) model, originally proposed by Chevalier \& Luo (1994) and studied numerically by Ròżyczka \& Franco (1996) and Garcia-Segura et al. (1999). In these models, an initially weak toroidal magnetic field is embedded in a radiatively driven wind. This configuration has been shown capable of accounting for a wide variety of outflow morphologies including highly collimated jets. These models clearly demonstrate the importance of magnetic fields. They cannot, however, account for the excess momentum in the flows (Bujarrabal et al. 2001) because of the weak fields which simply ride along in a radiatively driven wind.

The other class of model invokes so-called magnetocentrifugal launching (MCL; Blandford \& Payne 1982; Pelletier \& Pudritz 1992). This paradigm has, for many years, been explored as the mechanism driving jets in young stellar objects (YSOs), microquasars, and active galactic nuclei (AGNs). The MCL paradigm assumes the presence of a rotating central gravitating object (which may or may not include an accretion disk). In the case of a disk, plasma is threaded by a magnetic field whose poloidal component is in corotation with the disk. Disk-coronal gas is then subject to centrifugal force which accelerates the gas, flinging it out along field lines. The magnetized plasma eventually expands to a configuration where the toroidal component of the field dominates and hoop stresses collimate the flow. Thus, the MCL paradigm accounts for both the origin of the wind and the means of collimation. 
The success of the MCL paradigm in modeling jets associated with YSOs and AGNs has led some authors to suggest applying the idea in the context of PNs and PPNs (Blackman et al. 2001a; Frank \& Blackman 2004). Most recently it has been shown that the observed total energy and momentum in PPNs can be recovered with disk wind models using existing disk formation scenarios via binary interaction (Frank \& Blackman 2004 and references within).

Most theoretical investigations of the MCL paradigm assume a steady state flow. Observations suggest, however, that acceleration times for the flows are as much as an order of magnitude shorter than typical kinematical PPN ages (Bujarrabal et al. 2001). This implies that the mechanism responsible for the observed flows may operate explosively; i.e., the time over which the mechanism acts is short compared to the lifetime of the flow. Moreover, it has been suggested by Alcolea et al. (2001) that such a scenario would also provide the most straightforward explanation for the "Hubble law" kinematics observed in some PPN outflows (Balick \& Frank 2002; Bujarrabal et al. 1998; Olafsson \& Nyman 1999). The MCL paradigm can act transiently, however, when linked with the rapid evolution of its source, as for example in the case of the proposed mechanisms for gamma-ray bursts (GRBs; Piran 2005) and supernovae (SNe). This scenario has been investigated by a number of authors (Kluźniak \& Ruderman 1998; Wheeler et al. 2002; Akiyama et al. 2003; Blackman et al. 2006). In these scenarios differential rotation twists an initially weak poloidal field thereby generating and amplifying a toroidal field. When the toroidal component reaches a critical value it drives through the stratified layers of the collapsing core carrying trapped material with it. The hoop stresses associated with such a field also serve to collimate the flow.

Recently Matt et al. (2006) examined numerically a simplified version of this idea which was originally suggested for PPNs in Blackman et al. (2001b). In these studies the authors began with a gravitating core threaded by an initially poloidal field set rotating at $10 \%$ of the escape speed within an envelope of ionized gas. As the simulation progressed, the resulting toroidal field was sufficiently strong to drive a complete and rapid expulsion of the gaseous envelope. Since the initial conditions assumed in Matt et al. (2006) are applicable to either a young PPN or a collapsing protopulsar, it is reasonable to ask whether such transient events are occurring in the early stages of the formation of PNs, and if such events can serve as well as steady state jets can in accounting for the complex morphologies observed in such systems. We note that these classes of model are sometimes referred to as "magnetic towers" or "springs" because it is the gradient of toroidal field pressure that drives the outflow. Again we note that the magnetic fields needed for our scenario can be delivered by binary interactions as has been demonstrated by Nordhaus \& Blackman (2006) and Nordhaus et al. (2007).

There is growing evidence to suggest that magnetocentrifugal launch models are appropriate for PNs and, more importantly, PPNs (Vlemmings et al. 2006). Taking this evidence together with that for short acceleration times mentioned above $\left(\tau_{\text {acc }}<0.1 \tau_{\text {dyn }}\right)$, we argue that many PPNs may be shaped by shells which fragment into clumps rather than multiple jets.

There is a subset of PPNs and young PNs with multiple lobes of roughly similar size. These include CRL 2688, CRL 618, IRAS $19024+0044$, IRS 09371+1212, M1-37, and He 2-47. It is natural to try to interpret these structures initially as resulting from the action of jets. However, consideration of magnetocentrifugal launching models shows this to be unlikely. In all forms of the model, gravitational binding energy is tapped via rotational motions of the central source about some axis $\boldsymbol{\omega}$ and is converted into outflow kinetic energy using the magnetic fields as a "drive belt." The existence of a quasi-stable rotational axis is a requirement of the models in order to produce a continuous outflow. Multiple jets of equal length are difficult to imagine in such a scenario as the jets would then each require their own rotational engine with separate alignments. Even so-called magnetic tower models that drive the jet by winding up an initially weak poloidal field require a net spin axis such that $B_{\phi} \sim 2 \pi n_{\phi} B_{p}$, where $n_{\phi}$ is the number of turns about $\boldsymbol{\omega}$.

The production of multiple bow shocks from clumps or bullets driven by a transient MCL process is not as difficult to envision. In Matt et al. (2006), it was shown that the static envelope or atmosphere of a star could be entirely driven off of a rotating magnetized core. These models relied on the magnetic tower "spring" mechanisms, and the envelope becomes compressed into a thin shell which rides at the front of the expanding magnetic tower. Such a thin accelerating shell would be subject to a variety of instabilities including the Rayleigh-Taylor, thin shell (Vishniac 1983), and nonlinear thin Shell (Vishniac 1994) modes, all of which would be modified by the presence of an ordered magnetic field, which would impose a long coherence length onto the resulting flow. The precise details of such fragmentation in this situation have yet to be calculated and stand as an open problem. Given the impulsive acceleration of a dense, radiatively cooling shell into a lower density environment, it is likely that the shell would fragment into a number of high Mach number clumps directed along the poles (and perhaps the equator-see CRL 2688; Matt et al. 2006). A potential challenge to this model would be the creation of fragmentation modes that can, in some cases, produce roughly equivalent clumps in terms of propagation direction on either side of the source, as is already seen. Given that caveat, however, the ability for explosive magnetic tower models already explored in the literature to drive unstable shells makes them an attractive means of producing high Mach number clumps. As we have shown, these clumps, propelled into the surrounding media, then drive bow shocks which do at least as good a job as, if not better than, jets in recovering gross morphological and kinematic observations. Similar conclusions have recently been reached by Raga et al. (2007).

In summary, the results presented here add weight to an emerging paradigm in which transient (explosive) MCL processes act as the driver for PPN evolution in some cases. The fact that such magnetic launch mechanisms are already favored by some theorists to explain supernovae and gamma-ray bursts (Piran 2005) makes all the more compelling the notion that lower energy analogs of the processes believed to be occurring during the penultimate stages of massive stars' evolution are also occurring in low-and intermediate-mass stars.

The authors thank Alexei Poludnenko, Orsola De Marco, Joel Kastner, Patrick Hartigan, and Pat Huggins for their insights. We also thank the anonymous referee whose careful reading of our manuscript helped to improve this paper.

This material is based on work supported by NASA under award NM0710076, through Jet Propulsion Laboratory Spitzer Space Telescope Theory grant 051080-001; by the National Science Foundation under award AST 05-07519, and Hubble Space Telescope theory grant 11251; and by the Department of Energy under award DE-FC52-92SF19460 


\section{APPENDIX}

\section{THE COOLING PARAMETER AND THE RADIATIVE REGIME}

Following Blondin et al. (1990), we define the cooling parameter $\chi$ of an outflow according to

$$
\chi=\frac{d_{\text {cool }}}{d_{\text {hydro }}},
$$

where $d_{\text {hydro }}$ is a characteristic dynamical length which is taken to be the radius of the jet in Blondin et al. (1990), and which we take to be the radius of either the jet or the clump depending on which is under consideration. In our simulations this radius is $500 \mathrm{AU}$ for both clump and jet. The other length scale in this expression is the cooling length $d_{\text {cool }}$, which is essentially the thickness of the region behind the jet/clump shock where cooling occurs. For a radiative outflow, this length is small compared to the characteristic dynamical length for the flow. Thus, we expect that for radiative outflows we have $\chi \ll 1$.

We can explicitly follow the size of the cooling layers in our simulations by looking at plots of temperature. In Figure 11, we present one plot each for the clump (left) and the jet (right) cooling layers along the axis of the flow. These plots are taken at late times $(\approx 1000 \mathrm{yr})$ and represent upper limits on the size of the cooling layers. For most of the run time of our simulations, the cooling layers are actually found to be substantially narrower than those seen in Figure 11. If we characterize the width of the cooling layer using the full width at half-maximum (FWHM) as determined from these plots, we find for our upper limit on $\chi$ :

$$
\chi \lesssim 1
$$

We conclude that these simulations are solidly in the radiative regime with $\chi$ approaching 1 from below only at the very end of the runs.

To further support this conclusion, we next present a more theoretical argument showing that the flows should be and are in the radiative regime. In Figure 12 we present plots of velocity along the axis taken from the same data frames as those used in the plots of temperature. These figures indicate a postshock flow velocity, $v_{f}$, in the cooling layer of 79 and $86 \mathrm{~km} \mathrm{~s}^{-1}$ for the clump and jet, respectively. For a strong shock the shock velocity $v_{s}$ and $v_{f}$ are related according to

$$
v_{f}=\frac{3}{4} v_{s}
$$

We find $v_{s}=105 \mathrm{~km} \mathrm{~s}^{-1}$ for the clump and $v_{s}=115 \mathrm{~km} \mathrm{~s}^{-1}$ for the jet. This is the velocity behind the shock before cooling sets in. The corresponding postshock temperatures can be found from

$$
T_{s}=\frac{3 \mu m_{\mathrm{H}}}{16 k_{\mathrm{B}}} v_{s}^{2},
$$

for $2.5 \times 10^{5}$ and $3.0 \times 10^{5} \mathrm{~K}$ for clump and jet, respectively. These values are comparable to the maximum values seen in the plots of axial temperature in Figure 11.

The cooling length $d_{\text {cool }}$ can be expressed in terms of the cooling time $t_{\text {cool }}$ according to

$$
d_{\mathrm{cool}}=\frac{v_{s} t_{\mathrm{cool}}}{4}
$$
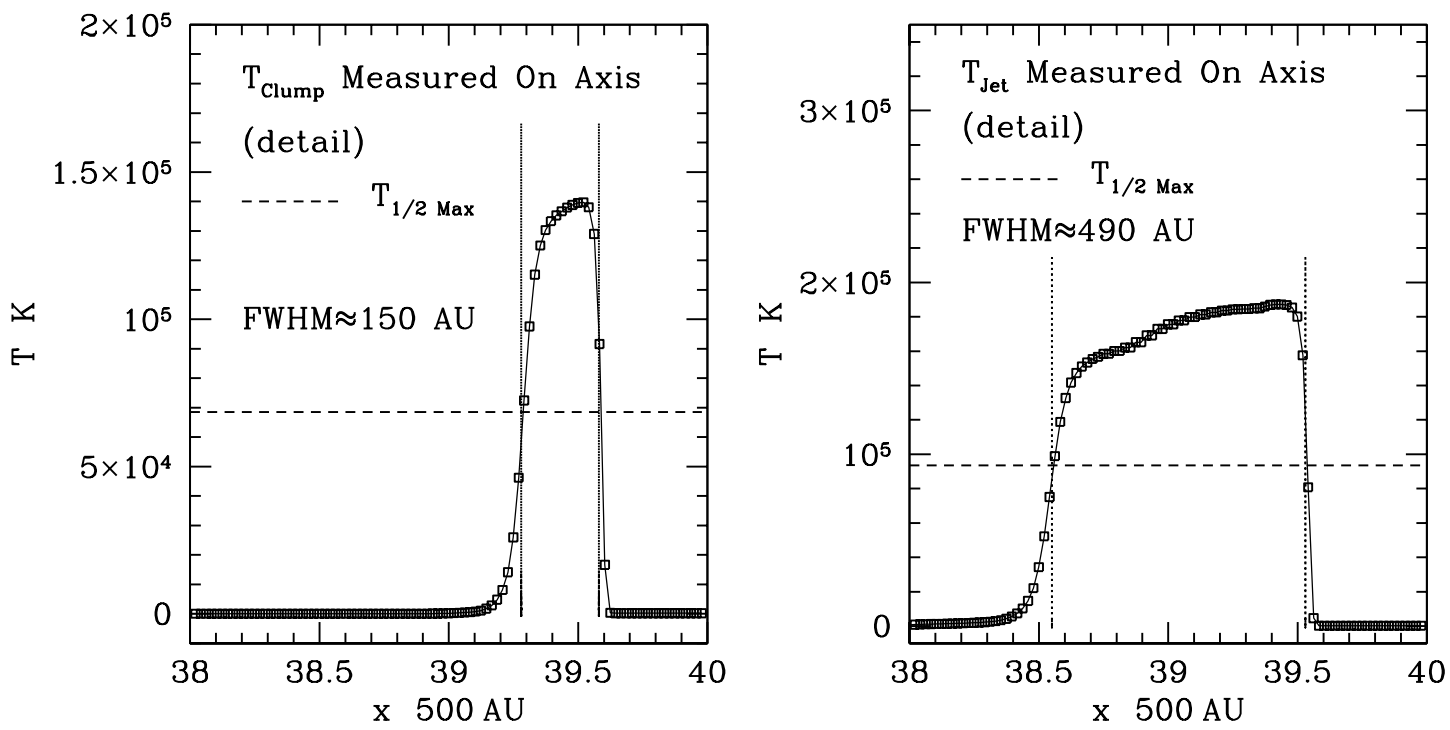

FIG. 11.- Temperature measure along symmetry axis for the clump (left) and jet (right) late in their respective simulations. 

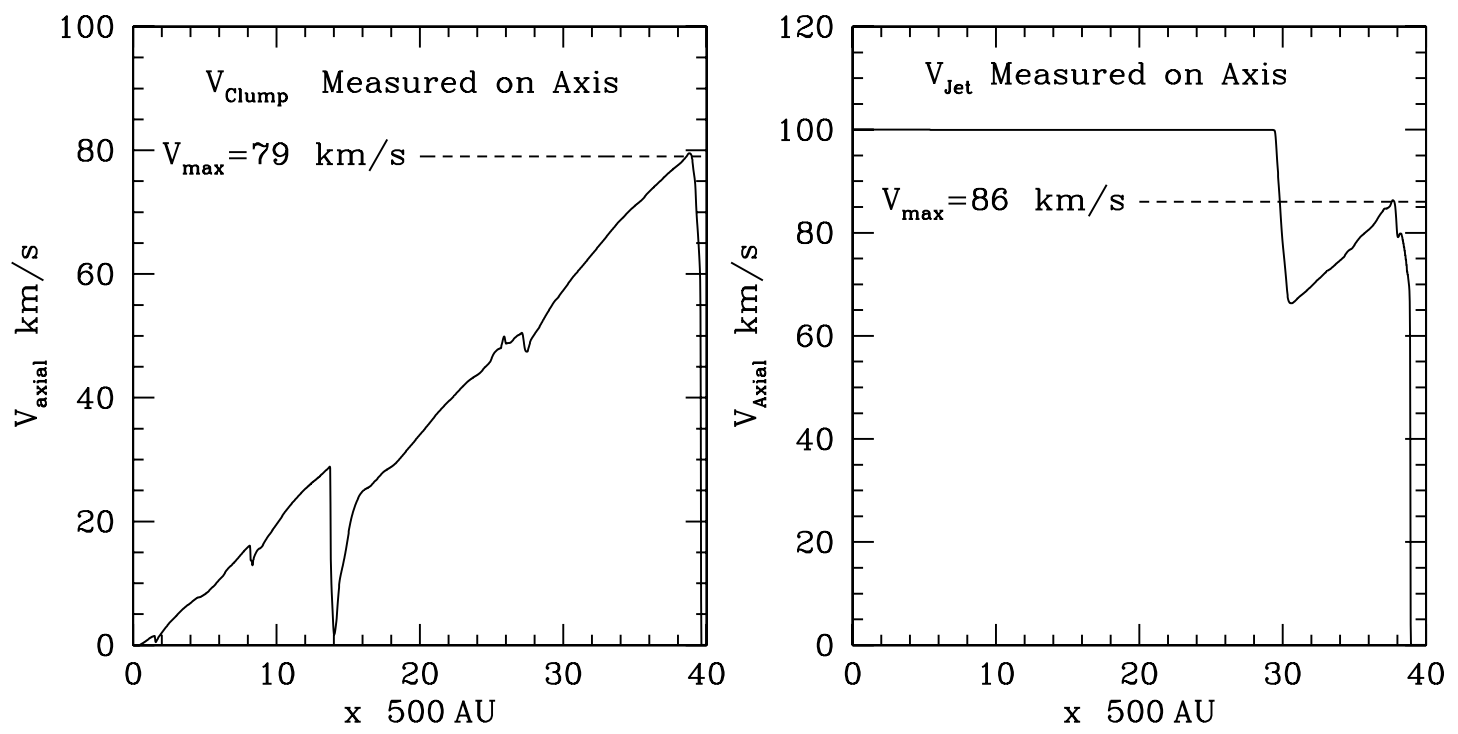

FIG. 12.-Velocity measure along symmetry axis for clump (left) and jet (right) late in their respective simulations.

where $t_{\text {cool }}$ is given by

$$
t_{\text {cool }}=\frac{k_{\mathrm{B}} T_{s}}{n_{0} \Lambda\left(T_{s}\right)} .
$$

The cooling function $\Lambda$ used in the simulations is the Delgarno-McCray cooling curve (Fig. 2 of Delgarno \& McCray 1972). From this figure we can see that in this temperature range $\Lambda \sim 10^{-21} \mathrm{erg} \mathrm{cm}^{3} \mathrm{~s}^{-1}$. These results imply a cooling time of $t_{\text {cool }} \sim 4 \mathrm{yr}$. Characteristic velocities in the problem are $\sim 10^{2} \mathrm{~km} \mathrm{~s}^{-1}$ Thus, we obtain a cooling length of $d_{\text {cool }} \sim 20$ AU and a cooling parameter of $\chi \sim 0.04$. This value is nearly 2 orders of magnitude smaller than the estimated upper limits based on the FWHMs determined from the plots of temperature. We can conclude that for most of the run time of our simulations the outflows modeled are well within the radiative regime and that they are still marginally radiative at the ends.

Furthermore, we note that in simulations run with higher density, the values of $t_{\text {cool }}, d_{\text {cool }}$, and more importantly $\chi$ would all decrease, placing our simulations deeper in the radiative regime. These simulations would therefore exhibit qualitatively similar morphological and kinematical features to the simulations already run. Thus, our simulations fully capture the fundamental characteristics of radiative bullets, and only the details of flow patterns such as the secondary, small-wavenumber instabilities will change as $\chi$ decreases further.

REFERENCES

Akiyama, S., Wheeler, J. C., Meier, D. L., \& Lichtenstadt, I. 2003, ApJ, 584 954

Alcolea, J., Bujarrabal, V., Sánchez Contreres, C., Neri, R., \& Zweigle, J. 2001, A\&A, 373, 932

Balick, B., \& Frank, A. 2002, ARA\&A, 40, 439

Blackman, E. G., Frank, A., Markiel, A. J., Thomas, J. H., \& Van Horn, H. M. 2001a, Nature, 409, 485

Blackman, E. G., Frank, A., \& Welch, C. 2001b, ApJ, 546, 288

Blackman, E. G., Nordhaus, J. T., \& Thomas, J. H. 2006, NewA, 11, 452

Blandford, R. D., \& Payne, D. G. 1982, MNRAS, 199, 883

Blondin, J. M., Bruce, A. F., \& Königl, A. 1990, ApJ, 360, 370

Borkowski, K. J., Blondin, J. M., \& Harrington, J. P. 1997, ApJ, 482, L97

Bujarrabal, V., Alcolea, J., \& Neri, R. 1998, ApJ, 504, 915

Bujarrabal, V., Castro-Carrizo, A., Alcolea, J., \& Sánchez Contreras, C. 2001, A\&A, 377, 868

Chevalier, R. A., \& Luo, D. 1994, ApJ, 421, 225

Cunningham, A. J., Frank, A., \& Blackman, E. G. 2006, ApJ, 646, 1059

Delgarno, A., \& McCray, R. A. 1972, ARA\&A, 10, 375

Fong, D., Meixner, A., Castro-Carrizo, A., Bujarrabal, V., Latter, W. B., Tielens, A. G. G. M., Kelly, D. M., \& Sutton, E. C. 2001, A\&A, 367, 652

Frank, A. 2006, in IAU Symp. 234, Planetary Nebulae in Our Galaxy and Beyond, ed. M. J. Barlow \& R. H. Méndez (Cambridge: Cambridge Univ. Press), 293

Frank, A., \& Blackman, E. G. 2004, ApJ, 614, 737

Garcia-Segura, G., Langer, N., Różyczka, M., \& Franco, J. 1999, ApJ, 517, 767
Icke, V. 2003, A\&A, 405, L11

Icke, V., Mellema, G., Balick, B., Eulderink, F., \& Frank, A. 1992, Nature, 355, 524

Kluźniak, W., \& Ruderman, M. 1998, ApJ, 505, L113

Lee, C.-F., \& Sahai, R. 2003, ApJ, 586, 319

LeVeque, R. J. 1997, J. Comput. Phys., 131, 327

Matt, S., Frank, A., \& Blackman, E. G. 2006, ApJ, 647, L45

Mellema, G., \& Frank, A. 1997, MNRAS, 292, 795

Nordhaus, J., \& Blackman, E. G. 2006, MNRAS, 370, 2004

Nordhaus, J., Blackman, E. G., \& Frank, A. 2007, MNRAS, 376, 599

Olafsson, H., \& Nyman, L.-Å. 1999, A\&A, 347, 194

Ostriker, E. C., Lee, C.-F., Stone, J. M., \& Mundy, L. G. 2001, ApJ, 557, 443

Pelletier, G., \& Pudritz, R. E. 1992, ApJ, 394, 117

Piran, T. 2005, Rev. Mod. Phys., 76, 1143

Poludnenko, A. Y., Frank, A., \& Mitran, S. 2004, ApJ, 613, 387

Raga, A. C., Esquivel, A., Riera, A., \& Velazquez, P. F. 2007, ApJ, 668, 310 Ròżyczka, M., \& Franco, J. 1996, ApJ, 469, L127

Sahai, R., \& Trauger, J. T. 1998, AJ, 116, 1357

Soker, N. 2000, MNRAS, 318, 1017

Trammell, S. R., Dinerstein, H. L., \& Goodrich, R. W. 1993, ApJ, 402, 249

Trammell, S. R., \& Goodrich, R. W. 2002, ApJ, 579, 688

Vishniac, E. T. 1983, ApJ, 274, 152 1994, ApJ, 428, 186

Vlemmings, W. H. T., Diamond, P. J., \& Hiroshi, I. 2006, Nature, 440, 58

Wheeler, J. C., Meier, D. L., \& Wilson, J. R. 2002, ApJ, 568, 807 